\title{
Proposição de reestruturação do sistema de gestão do Plano de Oferta de Cursos e Vagas do IFSC
}

\author{
Carla Cerdote da Silva \\ Fábio José Wojcikiewicz Caldas \\ Lilian Wrzesinski Simon \\ Rodolfo Amando Schmitz \\ Alexandre Moraes Ramos
}

\begin{abstract}
Mestranda em Administração Universitária. Universidade Federal de Santa Catarina (UFSC) Brasil - carlacerdote@gmail.com Mestrando em Administração Universitária. Universidade Federal de Santa Catarina (UFSC) Brasil - fjcalda@gmail.com Mestra em Administração Universitária. Universidade Federal de Santa Catarina (UFSC) Brasil - lilian.uffs@gmail.com Mestrando em Administração Universitária. Universidade Federal de Santa Catarina (UFSC) Brasil - rodolfoschmitz@gmail.com Doutor em Engenharia de Produção. Universidade Federal de Santa Catarina (UFSC) Brasil - amrrms@gmail.com
\end{abstract}

\section{RESUMO}

Este artigo tem como objetivo apresentar uma proposta de reestruturação do sistema de gestão do Plano de Ofertas de Cursos e Vagas (POCV) para a admissão de professores aplicados a todos os campi do Instituto Federal de Santa Catarina (IFSC), com vistas a adoção de uma nova plataforma, com uma modelagem informacional mais estruturada, funcional e alinhada aos seus objetivos. A metodologia partiu de uma abordagem qualitativa, baseada em fontes bibliográficas e documentais, associada à observação participante. Foram relacionados aspectos teóricos e normativos inerentes ao contexto em que o objeto se insere, para em seguida descrevê-lo, dimensionar suas funcionalidades, identificar suas limitações e mapear as adequações e adaptações necessárias ao desenvolvimento do sistema de gestão do POCV, com a estruturação do seu banco de Dados (BD) no propósito de superar as deficiências detectadas. $O$ estudo foi realizado durante o primeiro e segundo semestres de 2016. Esta nova modelagem é apresentada para trazer maior confiabilidade dos dados e segurança nas informações, a integração a todos os campi de forma simultânea e on-line, a padronização da forma de acesso ao sistema, racionalização do tempo para sua operacionalização, a flexibilidade de utilização pelo servidor responsável pela gestão do plano, facilidade de alimentação pelos usuários em plataforma amigável e autoexplicativa. Espera-se que o sistema possa contemplar as necessidades da instituição e superar as limitações reveladas no formato atual.

Palavras-chaves: Gestão Acadêmica e Administrativa. Sistema de Informações Gerenciais. Gerenciamento de Dados.

\section{Proposal for restructuring the management system of the plan for course offerings and vacancies at the IFSC}

\begin{abstract}
The objective of this article is to present a proposal for restructuring the management system of the Program of Offers of Courses and Vacancies (POCV) for the admission of teachers, which is applied in all Campuses of the Federal Institute of Santa Catarina (IFSC), with a view to adopting a new platform, with an informational modeling and that is also more structured, functional and in line with its objectives. The methodology was of a qualitative approach, based on bibliographic and documentary sources, associated with participant observation. The theoretical and normative aspects inherent to the context where the object is inserted are presented so as to describe it, to dimension its functionalities, to identify its limitations and to map the adaptations necessary to the development of the POCV management system, with the structuring of the database (BD) in order to overcome the detected deficiencies. The study was conducted during the first and second semesters of 2016. This new modeling is presented to bring greater reliability of data and information security, integration to all campuses simultaneously and online, standardization of the access to the system, rationalization of the time for its operationalization, flexibility of use by the server responsible for the management of the plan, as well as facility of feeding by users on a friendly and self-explanatory platform. It is expected that the system can address the needs of the institution and overcome the limitations revealed in the current format.
\end{abstract}

Keywords: Academic and administrative management, Management information system, Data management. 


\section{INTRODUÇÃO}

Com a revolução da informação, as organizações passaram a mudar seu comportamento, remodelando seus processos e inovando em seus modelos de gestão. A gestão de pessoas tornou-se então uma estratégia determinante para o sucesso dos negócios (DUTRA, 2009), ao mesmo tempo em que a maximização do uso da tecnologia passou a ser vista como uma forma de potencializar a gestão da informação nos mais diversos segmentos organizacionais (LAUDON; LAUDON, 2010; MANÃS, 2010). Explorar as potencialidades das pessoas com o uso da tecnologia também é uma necessidade das organizações públicas e nas Instituições de Ensino Superior (IES), que estão buscando gradativamente alinhar o formato de seus processos para poderem explorar as ferramentas tecnológicas que estão ao seu dispor.

Neste contexto, as Instituições Federais de Ensino Superior (IFES) e Institutos Federais de Educação, Ciência e Tecnologia (IFETs) são reconhecidos como partícipes de áreas estratégicas para o desenvolvimento do país, por sua alta especificidade nas diferentes áreas do saber e precisam estar aptos a gerir seus recursos, extraindo a sua máxima eficiência, para que possam agir proativamente frente aos desafios da gestão.

Essas instituições passaram, no decorrer dos anos, por mudanças relacionadas a sua forma de gestão, intensificando a busca por resultados nas mais diversas áreas, incluindo a política de gestão de pessoas. O cenário atual, altamente competitivo, globalizado e em expansão tecnológica exige a alocação adequada de pessoas, com o propósito de atender de forma satisfatória as necessidades da sociedade e do usuário final dos serviços prestados (GRILLO, 2001; DUTRA, 2009).

A publicação da Lei no 12.677/2012 e do Decreto n 7.312/2010, através do Ministério da Educação (MEC) instituiu e regulamentou uma nova política de controle na gestão de pessoas das IFES tornando possível a reposição imediata de pessoal em caso de vacâncias, sem autorização específica, respeitando o valor de referência pré-definido pelo Ministério do Planejamento e Gestão (MPOG) para cada entidade.

Desse modo, nos Institutos Federais (IFs), e dentre estes, no Instituto Federal de Santa Catarina (IFSC), cenário desta abordagem, foi instituído o Banco de Professor-Equivalente (BPE) de educação básica, técnica e tecnológica, em consonância com o Decreto no 7.312/2010 (BRASIL, 2010). Com a distribuição de vagas para a composição dos BPEs, por meio da Lei no 12.677/2012 (BRASIL, 2012), o IFSC adotou o Plano de Oferta de Cursos e Vagas (POCV) para nortear suas decisões acerca da oferta de cursos e vagas para a admissão de professores (IFSC, 2016c).

A gestão do sistema de gestão utilizado na contratação de novos professores é feita através da utilização de planilhas com o formato .ods (Open Document Spreadsheet - Calc/Libreoffice'), onde os dados são inseridos e manipulados manualmente. Como esse formato apresenta fragilidades, pois os dados ficam vulneráveis a comandos que podem prejudicar a integridade das informações, foi observado ao longo do período de utilização deste sistema, por um servidor técnico administrativo, que também é um dos autores desta proposta, a necessidade de sua transferência para um novo formato, que permita uma operacionalização mais segura e eficiente.

Portanto, o objetivo deste artigo é apresentar uma proposta de reestruturação do sistema de gestão do Plano de Ofertas de Cursos e Vagas (POCV) do IFSC, com vistas à adoção de uma nova plataforma, com uma modelagem informacional mais estruturada, funcional e alinhada aos objetivos deste plano. De acordo com o Plano de Desenvolvimento Institucional (PDI) do IFSC, para o período de 2015 a 2019, o POCV está relacionado aos seguintes objetivos:

A1 - Atender os potenciais alunos, considerando seus diferentes perfis e o contexto social, ambiental e econômico da sua região e A7-Melhorar a qualidade da aplicação dos recursos públicos, que possuem metas e indicadores intrinsecamente relacionados à oferta de vagas e número de alunos (IFSC, 2015, s. p.).

De acordo com Laudon e Laudon (2010), o gerenciamento de dados e informações por meio do uso adequado da tecnologia da informação confere excelência operacional, com maior agilidade, maior eficiência e melhores resultados nos serviços fornecidos. Por conseguinte, adotar uma nova plataforma para a gestão do sistema POCV é uma forma de melhorar a operacionalização das atividades relacionadas ao dimensionamento do corpo docente no IFSC para atender os objetivos supracitados.

Nesta perspectiva foi realizado um levantamento bibliográfico contemplando conceitos teóricos sobre temas como tecnologia da informação, gestão de pessoas, Banco de Dados (BD) e Sistemas de Informações Gerenciais (SIGs), que embasaram a construção da metodologia e da sequência de ações necessárias para o desdobramento dessa proposição que contempla a contextualização do IFSC, a descrição do POCV e seu sistema de gestão, 
identificando as suas funcionalidades e limitações e, por fim, apresenta o delineamento estrutural de um novo modelo de sistema com viabilidade de aplicação prática.

\section{A TECNOLOGIA DA INFORMAÇÃO E A GESTÃO DE PESSOAS}

A informação é um dos bens mais valiosos nas organizações. Quase todas as atividades ou processos produzem informações e elas precisam ser gerenciadas. Um dos recursos mais modernos para seu gerenciamento e, consequente, armazenamento é o uso da Tecnologia da Informação (TI), onde elas podem ser interligadas, coletadas, armazenadas e disseminadas (LAUDON; LAUDON, 2010; MAÑAS, 2010).

De acordo com O'Brien (2011, p. 26), "espera-se que gerentes previdentes e profissionais das empresas estejam cientes dos problemas e oportunidades apresentados pelo uso da tecnologia da informação, bem como estejam aptos a enfrentar tais desafios administrativos de forma eficaz".

Com o crescente avanço tecnológico e a globalização, a busca e o aprimoramento das informações tornouse um dos principais objetivos nas organizações, sendo a tecnologia, um elemento-chave, que auxiliou no processo de diferenciação organizacional. Quando a tecnologia não é bem empregada e as informações não são devidamente compreendidas, gera-se um clima de incerteza que afeta o ambiente e as tomadas de decisões, comprometendo a estrutura organizacional, os processos decisórios e a gestão de pessoas (SACILOTTI, 2011).

Com a transposição da Era Industrial para a Era da Informação e do Conhecimento, a gestão de pessoas passou por desafios que exigiram um redesenho de suas estratégias (DUTRA, 2009). De acordo com Gil (2001), a gestão de pessoas destacou-se na década de 80 e a partir daí passou a enfrentar uma série de adversidades de natureza ambiental e organizacional. Isso se deve à globalização da economia, seguida da evolução das comunicações, do desenvolvimento tecnológico, da competitividade, dentre outros fatores também relevantes.

O papel do trabalhador se transformou sobremaneira, passando da simples execução de tarefas para ser portador do conhecimento organizacional, o que exige da organização uma gestão de pessoas estruturada com mecanismos eficazes de identificação clara das potencialidades, das lacunas e deficiências no seu quadro funcional (GIL, 2001). Desde então, a gestão de pessoas passou a desempenhar um importante papel dentro do planejamento estratégico das organizações, com esforços no sentido de implementar políticas mais amplas, que contribuam diretamente com o ambiente organizacional (FARIAS; GAETANI, 2002).

Adentrando no contexto das IFES, a adoção de uma nova política para a gestão de pessoas é reforçada pela sua complexidade, tornando de fundamental importância o aprofundamento do conhecimento específico nesta área. Os servidores docentes representam uma parte da sustentação dessas instituições e através de políticas adequadas é possível desencadear uma melhoria contínua na administração, propiciando o crescimento da qualidade nos serviços prestados (GRILLO, 2001).

Diante disso, as políticas de planejamento de pessoal docente são indispensáveis para o funcionamento satisfatório da IES, que encontram na TI uma ferramenta de suma importância para gerenciarem suas demandas de acordo com as especificidades que possuem. A implementação e uso da TI é considerada uma maneira de viabilizar novos arranjos organizacionais de gestão de pessoas, possibilitando um amplo acesso a informações e a funções personalizadas e relevantes para a administração das IFES.

A TI engloba todo software e todo hardware necessários para se atingir os objetivos organizacionais, (LAUDON; LAUDON, 2010), sendo um dos recursos mais eficientes no que tange à coleta, processamento, armazenamento, transmissão e disseminação dos dados e informações sobre as suas diversas áreas, incluindo a gestão de pessoas. A adoção de um BD adequado às finalidades institucionais é uma forma de alcançar seu gerenciamento culminando na tomada de decisões mais efetivas.

Para Turban, Rainer e Potter (2007, p. 4), "um banco de dados é uma coleção de arquivos ou tabelas relacionadas contendo dados". Os dados de um sistema de informação são organizados e armazenados por uma ampla variedade de tecnologias gerenciais, sendo um recurso vital que resulta em várias mudanças nas organizações modernas e sua administração eficaz e eficiente é considerada como parte integrante da estratégia organizacional, (O'BRIEN; MARAKAS, 2013).

Laudon e Laudon (2010) distinguem o conceito de BD entre visão física, que mostra como os dados são organizados e estruturados no meio de armazenamento físico, e visão lógica, onde são apresentados como seriam percebidos pelos usuários finais ou especialistas da empresa.

O gerenciamento de bancos de dados é projetado com base em uma estrutura específica de dados, reduzindo sua redundância e inconsistência, capazes de fornecer aos usuários finais acesso rápido e fácil a 
informações armazenadas, não havendo a necessidade da construção de arquivos isolados. Uma dessas estruturas é conhecida como BD relacional (REZENDE; ABREU, 2008; LAUDON; LAUDON, 2010; O'BRIEN, 2011).

O'Brien (2011) complementa pontuando que o gerenciamento de bancos de dados é responsável pelo uso adequado da tecnologia para o gerenciamento dos dados da organização, compreendendo o desenvolvimento e manutenção do dicionário de dados, projeto e monitoração do desempenho dos bancos de dados e aplicação de padrões para seu uso e segurança.

$\mathrm{O}$ BD é um dos componentes da $\mathrm{Tl}$, que se apresenta com um importante mecanismo de acesso à informação, sendo que um bom gerenciamento de seu uso, pode ser um grande diferencial na gestão organizacional e na tomada de decisões. Os sistemas de informação, por sua vez, são mais complexos e também devem ser analisados tanto na perspectiva tecnológica quanto do ponto de vista organizacional (LAUDON; LAUDON, 2010).

\subsection{Sistemas de Informações Gerenciais (SIG)}

Para O'Brien (2011, p. 06) "Sistema de Informação (SI) é um conjunto organizado de pessoas, hardware, software, redes de comunicação e recursos de dados que coleta, transforma e dissemina informações em uma organização". Oliveira (2002, p. 39), afirma que o "Sistema de Informação Gerencial (SIG) é o processo de transformação de dados em informações que são utilizadas na estrutura decisória da empresa, proporcionando, ainda, a sustentação administrativa para otimizar os resultados esperados".

A tecnologia e os sistemas de informações são os principais recursos que a empresa dispõe para criar e inovar em seus produtos e serviços, sendo que os SI se tornaram essenciais para as organizações do mundo contemporâneo, porque correspondem a sinônimos de excelência operacional, novos produtos, serviços e melhor tomada de decisão. Das ferramentas que os administradores dispõem, a tecnologia e os SIs estão entre os mais importantes para atingir altos níveis de eficiência e produtividade (LAUDON; LAUDON, 2010).

O SIG deve ser usado para transformar dados em informações que auxiliem a organização no seu processo decisório. Segundo Stair (1998, p. 278), “o propósito básico de um SIG é ajudar a empresa a alcançar suas metas, fornecendo a seus gerentes detalhes sobre as operações regulares da organização, de forma que possam a controlar, organizar e planejar com mais efetividade e com maior eficiência".

Quando a arquitetura de informações é inadequada, as informações acabam sendo insuficientes para o usuário mesmo que os dados estejam disponíveis. Mañas (2010, p. 151) destaca que são comuns as queixas sobre "a incompatibilidade entre a forma como é apresentada a informação e os procedimentos existentes na área usuária". Isso "obriga a uma transcrição e, às vezes, elaboração de complementações dos dados proporcionados automaticamente, para que possam ser úteis em seu destino final" (MAÑAS, 2010, p. 151).

$\mathrm{Na}$ utilização de sistemas informatizados, nota-se que o SIG se torna uma ferramenta de gestão indispensável, precisando ser adaptado e atualizado constantemente para o atendimento das necessidades da instituição. A reestruturação do sistema POCV, portanto, se faz necessária para uma melhor operacionalização das ações referentes às projeções do quadro docente, considerando a estrutura do IFSC em sua totalidade.

\section{METODOLOGIA}

Partindo do conhecimento dos pressupostos básicos acerca da estruturação e finalidade de um SIG e da percepção das nuances que envolvem a utilização do sistema de gestão do POCV no IFSC, buscou-se, por meio de uma pesquisa bibliográfica e documental, aprofundar a análise do BD até então empregado nas suas operações, que se apresenta em planilhas em formato .ods no aplicativo Calc/Libreoffice. O objetivo dessa análise foi propor a reestruturação desse $\mathrm{BD}$, mediante a transposição para uma nova plataforma com capacidade de contemplar as necessidades institucionais no que se refere à utilização das informações obtidas nesse local.

Esta pesquisa possui abordagem qualitativa e baseou-se em fontes bibliográficas e documentais, associadas à observação participante, realizada por um dos autores que atua na Diretoria de Gestão de Pessoas (DGP) do IFSC. Essa técnica foi fundamental para a compreensão do problema de pesquisa e a consecução da análise proposta (GIL, 2008). O estudo e reconhecimento do sistema foi realizado durante o primeiro semestre de 2016 e a proposta de reestruturação foi desenvolvida no segundo semestre do respectivo ano.

As informações que circulam no BD do sistema de gestão do POCV são alimentadas no âmbito de todas as unidades do IFSC, e gerenciadas pela Pró-reitoria de Ensino (PROEN), sendo utilizadas também pela Pró-reitoria de Desenvolvimento Institucional (PRODIN) e pela DGP, que subsidiam as decisões de planejamento e contratação de pessoal docente. 
Portanto, a análise do sistema seguiu as premissas do método estruturalista (LAKATOS; MARCONI, 1992), onde a contextualização de suas funcionalidades e limitações é feita a partir de cada área (campus) onde é alimentado, considerando que posteriormente os dados podem ser generalizados, de forma que a construção da proposta de intervenção no sistema comporte as expectativas de cada usuário no âmbito do IFSC como um todo.

A avaliação e o dimensionamento dessas variáveis culminaram na descrição de uma nova proposta de intervenção que poderá ser desenvolvida pelos profissionais que gerenciam o sistema de gestão do POCV no IFSC, caso despertem interesse. Portanto, essa pesquisa caracteriza-se como um estudo de caso com enfoque descritivo e de natureza aplicada, resultando na proposição de um produto final passível de desenvolvimento e utilização (VERGARA, 2013; YIN, 2001).

\section{INSTITUTO FEDERAL DE SANTA CATARINA (IFSC)}

O IFSC foi criado em Florianópolis por meio do Decreto n 7.566, de 23 de setembro de 1909, como Escola de Aprendizes Artífices de Santa Catarina. Após diversas alterações de status se expandiu para outras localidades no interior do Estado e passou a oferecer uma variada gama de cursos, inclusive superiores de graduação e pósgraduação. Sua configuração atual ganhou materialidade através da Lei n 11.892, de 29 de dezembro de 2008, a qual o transformou em Instituto Federal de Educação, Ciência e Tecnologia, instituição, responsável pela oferta de educação básica, profissional e superior (BRASIL, 2008).

O IFSC faz parte do plano de expansão da Rede Federal de Educação Profissional e Tecnológica e possui 22 campidistribuídos por todas as regiões de Santa Catarina, sendo que cada campus oferece cursos em diferentes áreas do conhecimento e níveis de ensino, com forte inserção também nas áreas de pesquisa e extensão. Para tanto, conta com um quadro funcional docente específico para o atendimento das demandas locais e das exigências legais.

\subsection{Gestão do Banco de Professor Equivalente (BPE)}

O Banco de Professor Equivalente (BPE) de educação básica, técnica e tecnológica dos IFs, foi disposto pelo Decreto $n^{\circ}$ 7.312, de 22 de setembro de 2010. Esse Decreto delega aos IFs, a instituição do BPE como instrumento de gestão de pessoal, influenciando na decisão de realização de concursos públicos, nomeações de cargo de professor efetivo da carreira do Ensino Básico, Técnico e Tecnológico (EBTT) e contratação de professores substitutos para suprir suas necessidades excepcionais, constituindo em um importante instrumento de gestão administrativa de pessoal dessas instituições.

Apesar de ser um ato discricionário, a realização do concurso público e nomeação docente estão condicionadas a existência de cargo vago e a disponibilidade após a soma dos índices previamente definidos no BPE, que é composto pela soma dos professores do magistério do ensino básico, técnico e tecnológico e dos professores titulares livres do ensino básico, técnico e tecnológico, efetivos, substitutos e visitantes. O gerenciamento do BPE considera um limite previsto para cada instituição, variando conforme a carga horária de cada professor.

O controle de atividades docentes é gerenciado por meio do Plano Semestral de Atividades Docentes (PSAD). Esse instrumento objetiva apresentar o planejamento semestral individual dos docentes da instituição, formando uma base de dados com informações detalhadas acerca do trabalho docente, que está diretamente relacionado a ofertas de vagas (IFSC, 2016a).

Nesse sentido, a Lei no 11.892/2008 define que no mínimo 50\% das ofertas de vagas para alunos sejam voltadas para o objetivo de "ministrar educação profissional técnica de nível médio, prioritariamente na forma de cursos integrados, para os concluintes do ensino fundamental e para o público da educação de jovens e adultos" (BRASIL, 2008), 20\% para "cursos de licenciatura, bem como programas especiais de formação pedagógica, com vistas na formação de professores para a educação básica, sobretudo nas áreas de ciências e matemática, e para a educação profissional" (BRASIL, 2008) e os 30\% restantes voltados para outros tipos de oferta.

Ademais, foi firmado o Termo de Acordo de Metas e Compromissos (TAM) entre o IFSC e MEC para fins de estruturação, organização e atuação dos IFs. Este acordo consiste na atribuição de 19 metas e compromissos ao IFSC que relacionam-se com a necessidade de revisão e reorganização dos cursos e estrutura acadêmicas, articulação da educação profissional científica e tecnológica e da educação superior com a educação básica e da graduação com a pós-graduação, eficiência, eficácia e economicidade institucionais, objetivando planejamento e atendimento de metas e objetivos a curto, médio e longo prazos em articulação com as ações dos IFs com as políticas e diretrizes de educação do país. Essas metas e objetivos são apresentados, por vezes, em indicadores numéricos, ora qualitativos. 
A vigência do presente termo será de 12 anos contados da data de sua assinatura que ocorreu no ano de 2010 (IFSC, 2010).

A normatização das atividades didático-pedagógicas do IFSC, consta no Regulamento Didático Pedagógico (RDP) aprovado na Resolução $n^{\circ}$ 41/CONSUP/2014, que versa sobre toda a atividade acadêmica do instituto, desde a inscrição nos processos seletivos até a emissão de certificados e diplomas. Esse regulamento seguiu os preceitos descritos na Lei de criação dos IFs, Lei no 11.892/2008, no TAM e demais normas do sistema federal de ensino. O RDP está em consonância com a missão do IFSC e com toda sua estrutura de planejamento, administrativa, de ensino, pesquisa e extensão (IFSC, 2015).

Para consecução dos objetivos e metas de educação, o governo federal distribuiu a força de trabalho nos IFs conforme as determinações da Lei no 12.677/2012, expandindo, assim, os BPEs existente nos IFs. O aumento de força de trabalho trouxe a necessidade do IFSC repensar a sua forma de gestão de vagas docentes para atingir as metas citadas. Portanto, foi necessário desenvolver uma ferramenta capaz de gerenciar a força de trabalho futura face ao quadro atual. Assim, seria possível apresentar o planejamento desta estrutura frente às metas e objetivos educacionais estipulados.

\subsection{O Plano de Oferta de Cursos e Vagas (POCV)}

Com a distribuição de vagas para a composição dos BPEs, notou-se a necessidade de adoção de uma ferramenta de gestão capaz de atender as prerrogativas legais no que diz respeito à contratação de novos professores e esteja relacionada com as metas de gerenciamento interno e externo do IFSC. Para isso foi instituído o Plano de Oferta de Cursos e Vagas (POCV).

O POCV é um plano de gestão que nasceu da necessidade de um gerenciamento com novas lógicas, voltadas ao desenvolvimento de estratégias de orientação institucional. Para sua elaboração foram consideradas como premissas a oferta total de vagas docentes do IFSC distribuída entre os seus cursos presenciais e à distância, bem como a projeção de oferta de novos cursos. A gestão da oferta de cursos e vagas por meio desse plano possibilita o acompanhamento eficiente de sua execução, em conjunto com a comunidade escolar, além de facilitar ao gestor a construção de cenários e a qualificação da tomada de decisão no ato do planejamento, em atendimento às metas externas e internas da instituição definidas no PDI (IFSC, 2015).

O POCV é gerido por uma planilha desenvolvida no aplicativo CalC/Libreoffice, associada a um conjunto de diretrizes alinhadas ao PDI do IFSC, que projeta resultados do quadro de professores por área, demanda por salas de aula, indicadores de distribuição da oferta por tipo de curso, relação aluno-professor e índice de eficiência (IFSC, 2015). Sua gestão é realizada a partir da compilação de várias planilhas, originadas em cada um dos campi, que ao serem compiladas resultam em um documento único, com os dados gerais acerca da oferta de cursos e vagas do IFSC, do qual é possível obter diversos relatórios.

Conforme pode ser observado nas figuras 1 e 2, o sistema de gestão do POCV é dividido em nove abas, sendo oito visíveis e uma oculta ${ }^{2}$. Dessas abas, duas são para preenchimento do usuário, uma de instrução, duas de relatórios, uma de cadastro, uma de registro de versões do sistema, uma de registro de siglas e uma de cálculos e fórmulas intermediárias aos relatórios, está oculta.

Inicialmente é recomendado ao usuário do sistema, lotado no campus, a leitura da aba instruções onde é indicado um roteiro de preenchimento das informações nas demais abas.

A figura 1 apresenta a indicação das abas que integram o sistema.

${ }^{2}$ As nove abas do sistema POCV são: instruções, entrada, base, resumo, class2, docentes, docentes-lista, versões e CH_áreas_semestres, sendo esta oculta. 
Figura 1 - Relatório da aba resumo do sistema POCV do campus Caçador do IFSC

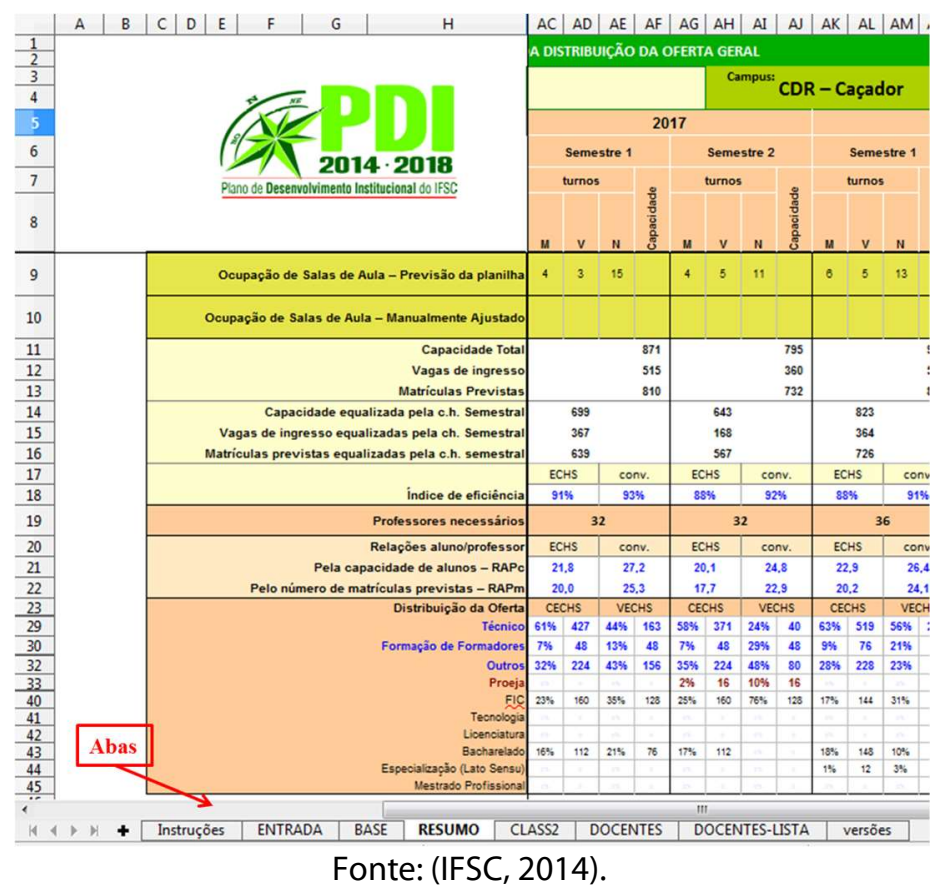

Prosseguindo na análise da figura 1, observa-se a aba "entrada", que é composta por dados oriundos de todos os campi do IFSC, sendo preenchida no quadro de entrada de cargas horárias por área de atuação dos professores. Isto significa adicionar os seguintes dados: nome do campus, dados do curso e das fases do curso; planejamento de entradas de turmas; planejamento de turmas para regime pleno; carga horária média semanal de referência; áreas de atuação e cargas horárias por área de atuação docente.

A aba base traz os dados contidos na aba entrada de forma compilada, contendo, ainda, cálculos intermediários à aba resumo. Por sua vez, a aba resumo, cujos dados são evidenciados na figura 1, é uma das abas de relatório, que traz a previsão de ocupação de salas, capacidade, vagas e matrículas previstas para a turma, os índices de eficiência, quantitativo de professores necessários para o semestre, relação aluno e professor e a distribuição de ofertas de cursos. Estas informações são distribuídas em linhas. Há também colunas para a distribuição das informações referentes aos semestres subsequentes.

A aba docentes-lista é preenchida com a nominata de todos os professores e suas respectivas áreas de atuação. Essas abas fornecem juntas insumos para relatórios disponibilizados em outras abas.

Outra aba que permite a obtenção de relatórios é a docentes, na qual são relacionados os cursos e a carga horária docente necessária para execução do curso, assim como o dimensionamento dos professores para atender àquela demanda, conforme ilustrado na figura 2 .

Figura 2 - Relatório da aba docentes do sistema POCV do campus Caçador do IFSC.

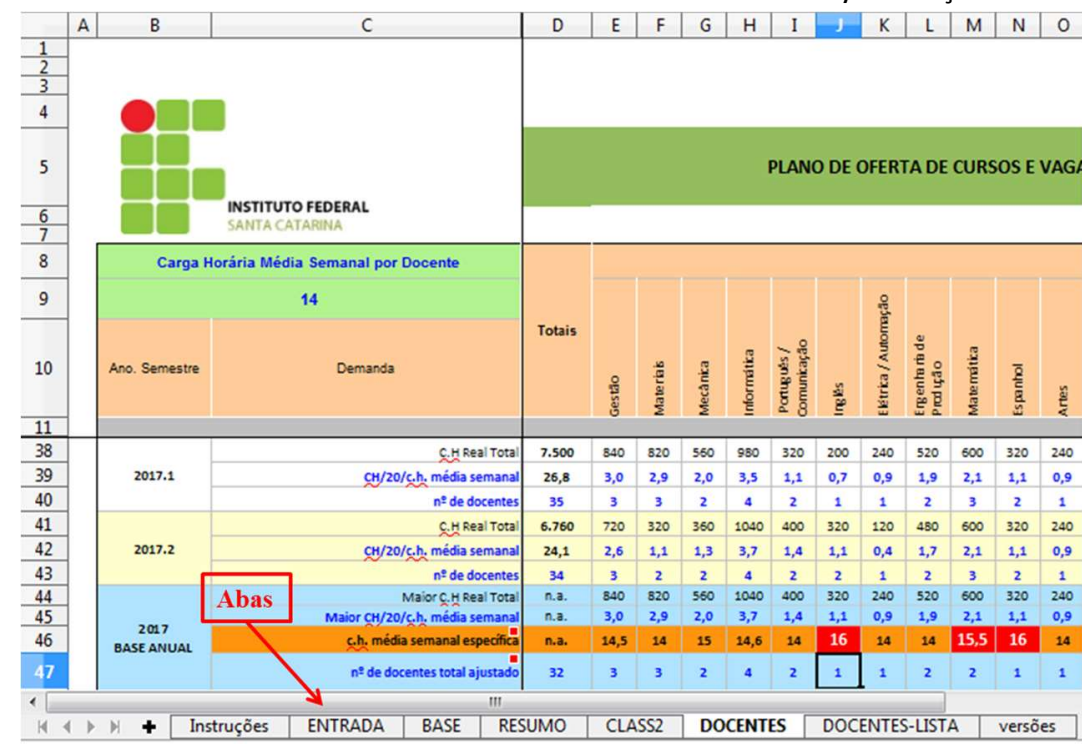

Fonte: (IFSC, 2014). 
Como pode ser visualizado na figura 2, se obtém como resultado um quadro intitulado docentes por área, a demanda por salas de aulas, os indicadores de distribuição da oferta por tipo de curso, a relação aluno-professor, índice de eficiência e a possibilidade de construção de cenários. Estas informações, aliadas ao planejamento institucional, subsidiam os gestores que utilizam o plano, lotados na PRODIN, na tomada de decisões, viabilizando o atendimento das metas internas e externas do IFSC.

A partir dessas constatações, percebe-se que o sistema de gestão do POCV foi construído para ser funcional, contudo apresenta fragilidades do ponto de vista operacional, estrutural e de integração e sistematização de informações.

$\mathrm{Na}$ aba instruções são detectadas orientações e detalhamentos de preenchimento, com o objetivo de indicar ao usuário em qual campo ou célula as informações devem ser dispostas. Não há, portanto, layout que permita ao usuário preencher os dados evitando a eminência de erros, com indicações da função de cada campo no decorrer do seu preenchimento. $O$ exemplo a seguir foi extraído do sistema POCV:

Informar dados do curso. Colunas B a L. [...] cada linha representa uma fase ou módulo semestral do curso. Por exemplo, um curso de 4 semestres requer 4 linhas de preenchimento, repetindo-se as informações das colunas de B a F. [...] O preenchimento das informações nas colunas de B a F deve ser feito exclusivamente via lista de seleção [...] (IFSC, 2014).

Estas informações estão em aba diversa da entrada e docentes, fazendo com o que o usuário tenha que percorrer vários caminhos entre essas abas para seguir todos os passos corretamente. Ainda, conforme a versão instalada do LibreOffice Calc, as características de preenchimento podem variar.

Observando o gerenciamento das tabelas, verifica-se que a integração entre os dados é realizada com o envio das planilhas dos campià PROEN, onde os dados são compilados por meio de tabelas dinâmicas e macros. Os arquivos são preenchidos e armazenados no computador do usuário, lotado no campus, para posteriormente serem encaminhados ao gestor, na reitoria. Caso haja a adição de dado incorreto em algum campo ou a desconfiguração de alguma célula ou fórmula, é gerada uma inconsistência nos relatórios, havendo a necessidade do gestor do sistema apurar o erro para correção. A disponibilização dos arquivos para download é realizada de forma estática, sem as devidas alterações no decorrer da execução do gerenciamento do sistema do POCV.

Além das fragilidades estruturais da planilha e de suas tabelas, observa-se detalhes ergonômicos e de padronização que precisam ser melhorados, como a nomenclatura das abas, ausência de notas explicativas no decorrer do preenchimento, falta de indicação nas próprias células de quais devem ser preenchidas e quais geram resultados, levando o usuário à incerteza de que realizou o preenchimento correto de todos os requisitos do sistema. O sistema não aponta os erros ocorridos no processo de alimentação dos dados pelo usuário, o que gera falhas ocultas e alteração de dados e informações sem que o usuário perceba.

Essas possibilidades de inconsistências e erros humanos durante a operacionalização do processo, tendo em vista a necessidade de intervenção manual no processamento dos dados, justificam a implementação de um sistema mais seguro, padronizado e unificado.

Ademais, para a segurança e consistência dos dados, é necessário o armazenamento destes em local único, estruturado de forma que não dependa da transmissão de informações alimentadas em arquivos distintos, facilitando o gerenciamento, acompanhamento dos dados, independente da utilização pelos usuários. Isso evita que dados sejam danificados ou perdidos por adulterações ou até mesmo inconsistências de arquivos.

Esses fatores demonstram que apesar do modelo atual de sistema de gestão do POCV responder às necessidades da instituição, apresenta limitações que o tornam ineficiente frente as demandas emergentes do IFSC, que continuou se expandindo, com a criação de novos campi e cursos após a adoção desse sistema na gestão do POCV. A dificuldade de operacionalização do sistema devido ao excesso de dados expostos na área de aplicação é um dos principais pontos observados com o crescimento institucional. Ademais, o formato em planilha em formato .ods (Open Document Spreadsheet - Calc/Libreoffice) deixa esses dados vulneráveis no que tange à segurança, comprometendo a confiabilidade das informações.

\subsection{Proposição de modelagem para o sistema}

Propõe-se a construção de um sistema com uma plataforma de modelagem relacional mais estruturada, operacional, funcional e alinhada aos objetivos do POCV, que esteja disponível online, centralize as informações de todos os campido IFSC e possibilite a integração ao PSAD. Com o sistema nessa nova plataforma, o gerenciamento, planejamento e acompanhamento institucionais ocorrem de forma unificada, conferindo maior segurança na 
manipulação dos dados e apresentação de indicadores concretos e funcionais para a tomada de decisão. Assim, seria possível a utilização constante pelos gestores, não somente durante o processo de planejamento como ocorre na versão atual, visto que com inputs externos e integração dos dados de todos os campi, sua atualização seria automática e instantânea.

A proposta de estrutura do sistema é baseada em um BD relacional, gerenciado através do sistema MySQL, disponível on-line (web). A escolha da utilização do MySQL deu-se por sua confiabilidade e facilidade no desenvolvimento de sistemas e administração dos dados, além de ser gratuito e compatível com todos os sistemas operacionais (MILANI, 2007).

Em princípio a modelagem do sistema seria dividida em seis domínios:

1. Campi: nominata campi, eixos, cursos, códigos da disciplina (incluindo fase e semestre), modalidades, semestres de oferta, carga horária necessária por área para cada turma (por período).

2. Códigos cursos e carga horária: curso, código da disciplina, semestre, fase, carga horária requerida por área, professor vinculado à disciplina.

3. Cursos: capacidade, formas e periodicidade de ingresso, código da disciplina.

4. Professores: matrícula SIAPE, nome, áreas de atuação, código da disciplina em que atua, carga horária, por semestre.

5. Oferta de vagas discentes: estimativa de oferta de vagas discentes, por código de disciplina.

6. Dados capacidade física dos campri. descrição das salas físicas, capacidade, turnos, código da disciplina de ocupação.

Destaca-se que estes domínios, após a primeira normalização, produzem a estrutura de tabelas apresentadas na figura 3. Todavia, após a segunda e terceira normalizações, a fim de implantar o BD, certamente surgirão novas tabelas visando unir as funcionalidades requeridas ao sistema. A figura 3 apresenta a nova estrutura proposta para o $\mathrm{BD}$, sem demonstrar os relatórios gerados pelo sistema, visto ser apenas o primeiro escopo da plataforma.

Figura 3 - Proposta de domínios em BD em MySQL.

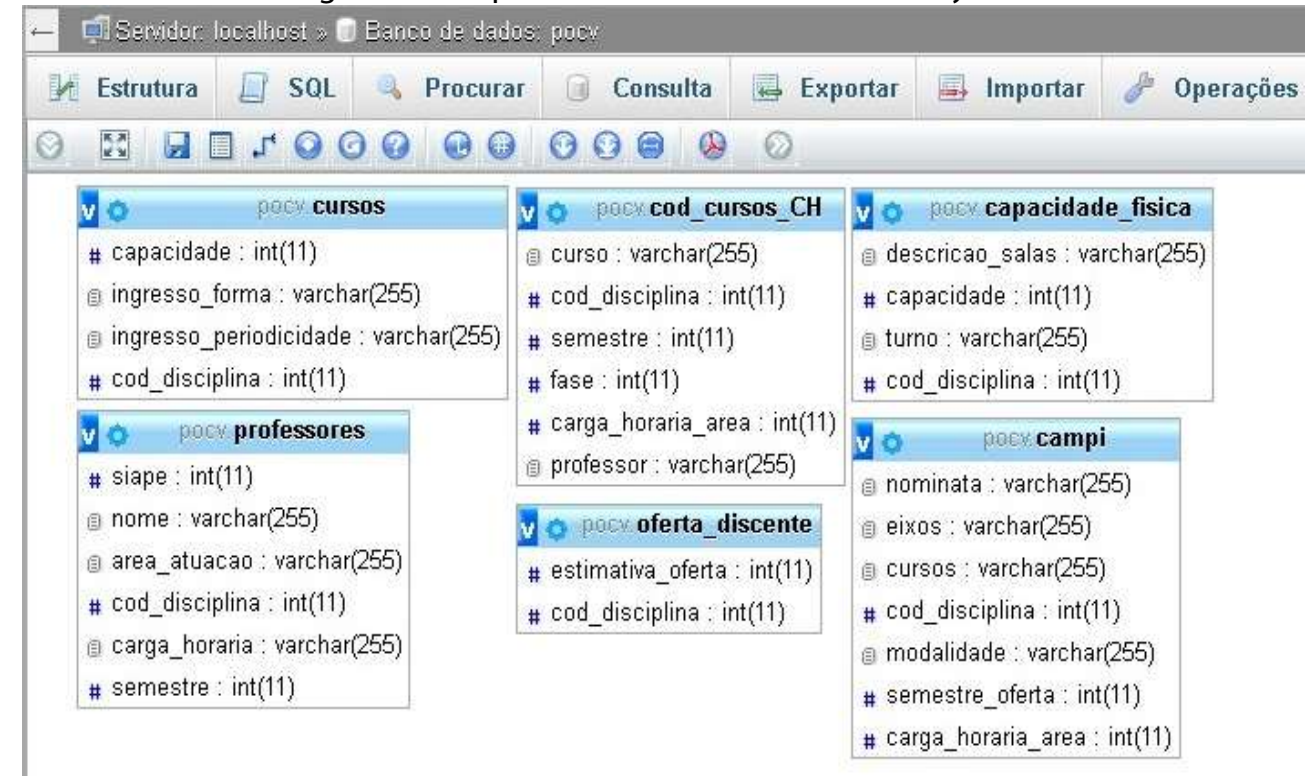

Fonte: Elaborado pelos autores (2016).

Com base nesta arquitetura de BD é possível integrar os dados de todos os campi do IFSC de maneira unificada, segura, padronizada e sem redundâncias, com consequente ampliação das possibilidades de emissão de relatórios gerenciais, permitindo o acesso simultâneo e instantâneo a todos os usuários dos campie aos gestores do sistema na reitoria, nos moldes sugeridos por Rezende e Abreu (2008).

Do cruzamento desses dados serão obtidos relatórios referentes à ocupação de salas de aulas, capacidade dos campide execução dos cursos, número de alunos necessários para atingimento de metas, número de professores necessários por áreas de atuação e cursos e consequente indicação de necessidade de contratação de novos professores por área, relações entre alunos e professores, carga horária média anual de professor, alcance de metas internas e externas do IFSC, ficando a cargo do sistema a correlação entre os dados inseridos e a avaliação destas metas, sendo mais um indicativo na tomada de decisão.

Com a disponibilidade de relatórios de forma segura, instantânea e dinâmica, e com a análise automatizada das metas internas e externas do IFSC, os gestores poderão agir com mais convicção, resguardados em parâmetros seguros e atualizados constantemente, o que permite a projeção de um cenário mais próximo da realidade. 


\section{CONCLUSÃO}

Esse artigo aborda os mecanismos de gestão de pessoal docente que vigoram no IFSC considerando sua atuação a nível estadual, de acordo com os preceitos regulamentares nacionais e as políticas institucionais estabelecidas para sua execução, apresentando uma proposta de reestruturação para o sistema de gestão do POCV, com vistas à adoção de uma nova plataforma, com uma modelagem informacional mais estruturada, operacional, funcional, integrada e alinhada aos objetivos deste plano. A consecução desse objetivo embasou-se em uma abordagem bibliográfica caracterizando a importância da TI, BD e SIG nos processos de gerenciamento institucional e como fatores significativos nas mudanças organizacionais. Os aspectos teóricos e normativos apresentados previamente à descrição do sistema constituíram o pano de fundo para a compreensão da finalidade do sistema, identificação de suas funcionalidades e as limitações que precisam ser superadas.

Em seguida, o funcionamento do sistema de gestão do POCV foi descrito, com vistas à proposição da sua reestruturação, com uma nova modelagem que abarque as necessidades da instituição superando as limitações existentes. A apresentação da nova plataforma contempla um sistema com uma base de dados relacional capaz de fornecer informações sistematizadas e relatórios na área de aplicação.

Essa análise dedicou-se a propor melhorias nos mecanismos de gestão do sistema POCV. Considerando a estrutura atual do IFSC, entende-se que a transposição da planilha Calc/Libreoffice, utilizada atualmente, para um SIG com um BD distinto, gerenciado por meio de $\mathrm{MySQ}^{3}$, em um sistema disponível on-line (web) seja uma solução adequada e viável.

Após o seu desenvolvimento, esse sistema apresentará entre suas vantagens, maior confiabilidade dos dados e segurança das informações, a sua integração a todos os campide forma simultânea e on-line, padronização da forma de acesso ao sistema sem depender de versões de software legados instalados no computador do gestor, racionalização do tempo para sua operacionalização, flexibilidade de utilização pelo servidor responsável pela gestão do plano, facilidade de alimentação pelos usuários em plataforma amigável e autoexplicativa.

O presente ensaio apresentou uma proposta inicial com uma nova modelagem de dados para a estruturação de um BD capaz de solucionar as deficiências detectadas. Esse novo BD visa contemplar as necessidades da instituição e superar as limitações reveladas no formato atual. Para o desenvolvimento completo do sistema é necessária uma análise mais aprofundada sobre os requisitos para a integração com o sistema PSAD, com mapeamento detalhado dos campos necessários e sua formatação. Ainda, cabe a consulta aos gestores do sistema visando a definição de layout apropriado e ampliação do sistema além do objeto desse estudo.

As ações propostas nessa pesquisa também podem ser ampliadas com o detalhamento do processo de integração do sistema PSAD à nova plataforma do sistema de gestão do POCV, visando à automatização dos inputs de dados docentes. Isso resultaria na inserção automática destas atividades no sistema de gestão do POCV, reduzindo a necessidade de alimentação manual no campus, e consequentemente, otimizando o fluxo de tarefas.

Como a proposição desse trabalho compreende somente a primeira normatização do sistema, é prudente que sejam propostas novas normatizações e criados novos domínios, observada a necessidade durante a sua implantação. A definição e detalhamento das relações entre os domínios para elaboração de relatórios do sistema POCV precisa estar atrelada ao processo de tomada de decisão. Portanto, cabe a consulta e demonstração dessas possibilidades à gestão do IFSC para a criação de novos modelos de relatórios, adaptados para o fornecimento das informações consideradas importantes para o processo decisório.

A reestruturação desse sistema é apresentada como um diferencial de inovação para o IFSC, e sua implementação é significativa no seu gerenciamento administrativo, com a padronização de acesso e a redução de erros e retrabalho. Acredita-se que sendo uma ferramenta confiável para a tomada de decisão, controle e informação, também poderá servir de modelo para a implantação nos IFs de todo o Brasil.

\section{REFERÊNCIAS}

BRASIL. Lei no 11.892 de 29 de dezembro de 2008. [2008]. Institui a Rede Federal de Educação Profissional, Científica e Tecnológica, cria os Institutos Federais de Educação, Ciência e Tecnologia, e dá outras providências. Disponível em: <http://www.planalto.gov.br/ccivil_03/_ato2007-2010/2008/lei/l11892.htm>. Acesso em: 13 jun.

${ }^{3}$ MySQL é um sistema de gerenciamento de banco de dados (SGBD), que utiliza a Linguagem de Consulta Estruturada - SQL. (MILANI, 2007) 
2016.

BRASIL. Decreto $\mathbf{n}^{\circ} \mathbf{7 . 3 1 2}$ de 22 de setembro de 2010. [2010]. Dispõe sobre o banco de professor equivalente de educação básica, técnica e tecnológica, dos Institutos Federais de Educação, Ciência e Tecnologia vinculados ao Ministério da Educação, e dá outras providências. Disponível em: <http://www.planalto.gov.br/ccivil_03/_Ato20072010/2010/Decreto/D7312.htm>. Acesso em: 13 jun. 2016.

BRASIL. Lei no 12.677 de 25 de junho de 2012. [2012]. Dispõe sobre a criação de cargos efetivos, cargos de direção e funções gratificadas no âmbito do Ministério da Educação, destinados às instituições federais de ensino; altera as Leis $\mathrm{n}$ os 8.168, de 16 de janeiro de 1991, 11.892, de 29 de dezembro de 2008, e 11.526, de 4 de outubro de 2007; revoga as Leis $n^{\circ}$ 5.490, de 3 de setembro de 1968, e 5.758, de 3 de dezembro de 1971, e os Decretos-Leis $n^{\circ} 245$, de 28 de fevereiro de 1967, 419, de 10 de janeiro de 1969, e 530, de 15 de abril de 1969; e dá outras providências. Disponível em: <http://www.planalto.gov.br/ccivil_03/_ato2011-2014/2012/lei/L12677.htm>. Acesso em: 13 jun. 2016.

DUTRA, Joel Souza. Gestão de pessoas: modelos, processos, tendências e perspectivas. São Paulo: Atlas, 2009.

FARIAS, Pedro César Lima de.; GAETANI, Francisco. A política de recursos humanos e a profissionalização da administração pública no Brasil do século XXI: um balanço provisório. Lisboa: CLAD, 2002.

GIL, Antônio Carlos. Gestão de pessoas: enfoque nos papéis Profissionais. São Paulo: Atlas, 2001.

GIL, Antônio Carlos. Métodos e técnicas de pesquisa social. 6. ed. São Paulo: Atlas, 2008.

GRILLO, Antônio Niccoló. Gestão de pessoas: princípios que mudam a administração universitária. Florianópolis: UFSC, 2001

IFSC. Acordo de metas e compromissos para estruturação, organização e atuação dos Institutos Federais criados pela Lei n $\mathbf{1 1 . 8 9 2}$ de 29 de dezembro de 2008. [2008] Disponível em $<$ http://ararangua.ifsc.edu.br/site_old/docs/planejamento/TAM\%20\%20Termo\%20de\%20Acordo\%20de\%20Metas.pdf>. Acesso em: 11 ago. 2016.

Resolução no 41/2014/CONSUP, de 20 de novembro de 2014. [2014] Disponível em: <http://cs.ifsc.edu.br/portal/files/Consup2014/resolucao41comRDPeGLOSSARIO.pdf $\geq$. Acesso em: 26 ago. 2016.

Plano de Desenvolvimento Institucional - PDI 2015 - 2019. [2015] Disponível em: <http://pdi.ifsc.edu.br/download/faca-o-download-do-pdi-2015-2019/>. Acesso em 13 jun. 2016.

Atividades Docentes - PSAD. [2016a] Disponível em: <http://www.ifsc.edu.br/estatisticas-atividadesdocentes-psad>. Acesso em: 20 ago. 2016.

Portal Institucional do IFSC. [2016b] Disponível em: <http://www.ifsc.edu.br/menuinstitucional/missao?id=152>. Acesso em: 10 ago. 2016.

. Plano de Oferta de Cursos e Vagas. [2016c] Disponível em: <http://pdi.ifsc.edu.br/download/plano-deoferta/planilhas-dos-campus/planilhas-do-pocv-versao-6-2/>. Acesso em 10 jul. 2016.

LAKATOS, Eva Maria; MARCONI, Marina de Andrade. Metodologia científica. 2. ed. São Paulo. Atlas, 1992.

LAUDON, Keneth; LAUDON, Jane. Sistemas de Informações Gerenciais. 9. ed. São Paulo: Pearson Prentice Hall, 2010.

MAÑAS, Antonio Vico. Administração de sistemas de informação: como otimizar a empresa por meio dos sistemas de informação. 8. ed. São Paulo: Érica, 2010.

MILANI, André. MySQL - Guia do Programador. São Paulo: Novatec, 2007.

O'BRIEN, James A. Sistemas de informação e as decisões gerenciais na era da internet. 3. ed. São Paulo: Saraiva, 2011.

O'BRIEN, James A.; MARAKAS, George M. Administração de sistemas de informação. 15. ed. Porto Alegre: AMGH, 2013. 
OLIVEIRA, Djalma de Pinho Rebouças de. Sistemas de informação gerenciais: estratégias, táticas, operacionais. 8. ed. São Paulo: Atlas, 2002.

REZENDE, Denis Alcides; ABREU, Aline França de. Tecnologia da Informação Aplicada a Sistemas de Informação Empresariais. 5. ed. São Paulo: Atlas, 2008.

SACILOTTI, Adaní Cusin. A importância da tecnologia da informação nas micro e pequenas empresas: um estudo exploratório na região de jundiaí. 2011.166p. Dissertação (Mestrado em Administração) - Faculdade de Campo Limpo Paulista, São Paulo.

STAIR, Ralph M. Princípios de sistemas de informação. Rio de Janeiro: LTC, 1998.

TURBAN, Efrain; RAINER JR., R. Kelly; POTTER, Richard E. Introdução a sistemas de informação: uma abordagem gerencial. Rio de Janeiro: Elsevier, 2007.

VERGARA, Sylvia Constant. Projetos e relatórios de pesquisa em administração. 14. ed. São Paulo: Atlas, 2013.

YIN, Robert K. Estudo de caso: planejamento e métodos. 2. ed. Porto Alegre: Bookman, 2001. 